\section{Case Reports in Ophthalmology}

Case Rep Ophthalmol 2020;11:481-485

\begin{tabular}{l|l}
\hline DOI: $10.1159 / 000509341$ & (c) 2020 The Author(s)
\end{tabular}

Published online: August 11, 2020

Published by S. Karger AG, Basel www.karger.com/cop

This article is licensed under the Creative Commons Attribution-NonCommercial 4.0 International License (CC BY-NC) (http://www.karger.com/Services/OpenAccessLicense). Usage and distribution for commercial purposes requires written permission.

\title{
Acute Lymphoblastic Leukemia Presenting as Acute Vogt-Koyanagi-Harada Syndrome
}

\author{
Narges Hassanpoor ${ }^{a, b} \quad$ Mohamad Reza Niyousha $^{a}$ \\ aRetina and Vitreous Service, Nikookari Eye Hospital, Tabriz University of Medical \\ Sciences, Tabriz, Iran; ${ }^{b}$ Ophthalmic Research Center, Shahid Beheshti University of \\ Medical Sciences, Tehran, Iran
}

\section{Keywords}

Subretinal fluid - Acute lymphoblastic leukemia - Exudative retinal detachment . Lymphoma $\cdot$ Vogt-Koyanagi-Harada syndrome $\cdot$ Central serous chorioretinopathy

\begin{abstract}
We aimed to describe a case of acute lymphoblastic leukemia (ALL) that initially presented and was managed as Vogt-Koyanagi-Harada syndrome (VKH). A 62-year-old man was referred for vision loss starting 1 week ago. There was no prior systemic or ocular history. Visual acuity was 20/200 in both eyes. Anterior segment exam was also normal in both eyes. On funduscopy, foveal reflex was significantly reduced. On optical coherence tomography, subretinal fluid was evident in both eyes. Fluorescein angiography showed a hypofluorescent area compatible with subretinal fluid and multiple pinpoint hyper- and hypofluorescent dots surrounding the detached retina. After 4 days, we were informed that the patient had been admitted to a general hospital due to spontaneous ecchymosis and melena. On complete blood count, there was a high white cell count, thrombocytopenia, and low hemoglobin concentration with a probable diagnosis of leukemia. On bone marrow biopsy, ALL with B-cell precursor was confirmed. In conclusion, exudative retinal detachment can be a primary presentation of leukemia and/or lymphoma. In atypical VKH or acute central serous chorioretinopathy cases with multiple pinpoint leakages
\end{abstract}




\section{Case Reports in Ophthalmology}

\begin{tabular}{l|l}
\hline DOI: $10.1159 / 000509341$ & ( 2020 The Author(s). Published by S. Karger AG, Basel
\end{tabular} www.karger.com/cop

Hassanpoor and Niyousha: Acute Lymphoblastic Leukemia Presenting as Acute VogtKoyanagi-Harada Syndrome

distributed mostly in the periphery of the subretinal fluid area (not randomly scattered throughout the subretinal fluid), we should consider leukemia.

(C) 2020 The Author(s)

Published by S. Karger AG, Basel

\section{Introduction}

Retinal manifestations are the most common ocular signs in leukemia, and they are divided into primary or secondary. The first category is due to malignant cell invasion, and the second category is due to complications of the treatment, infections, or blood alterations. Some of the secondary retinal manifestations of acute lymphoblastic leukemia (ALL) include retinal hemorrhages, Roth spots, and retinal ischemic manifestations, such as vascular occlusions and scattered cotton wool spots [1]. Exudative retinal detachment is a rare manifestation in ALL [1]. There are very few reports of leukemia presenting initially with this ocular manifestation in the literature [2]. Here, we report a case of ALL in a 62-year-old man with Vogt-Koyanagi-Harada (VKH)-like presentation as primary manifestation of his leukemia. According to our literature review, this is the first case of ALL presenting with VKH-like syndrome in Iran.

\section{Case Report}

The patient was a 62-year-old man referred to our clinic with the chief complaint of decreased visual acuity starting 1 week ago. There was no prior systemic or ocular history. Visual acuity was 20/200 in both eyes. Intraocular pressure was $15 \mathrm{~mm} \mathrm{Hg}$ in his right eye and 17 $\mathrm{mm} \mathrm{Hg}$ in his left eye. On slit lamp examination, there was no conjunctival infection, the anterior chamber was normal, and there was mild cataract without significant refractive error in both eyes. There were trace cells visible in the anterior vitreous of both eyes. Optic disc was pink bilaterally with no atrophic changes. Foveal reflex was significantly reduced bilaterally. When we examined the patient, he was healthy with no significant other systemic complaints. On optical coherence tomography (OCT), multi-lobulated retinal detachments were evident in both eyes. We were not able to assess the exact choroidal thickness on OCT; however, compressed choriocapillaris and thicker than normal choroid were visible. Fluorescein angiography (FA) showed a hypofluorescent area compatible with subretinal fluid and multiple pinpoint hyper- and hypofluorescent dots within the detached retina. Hyperfluorescent dots were distributed mostly in the periphery of the subretinal fluid area while sparing the central macula (Fig. 1). Our first differential diagnoses were VKH and central serous chorioretinopathy (CSCR). There were no clear auditory or integumentary symptoms. There also was no history of recent headache or neck stiffness. Other differential diagnoses were lymphoma and choroidal metastasis due to the patient's age. Our patient's old age (more than 60 years) was not typical for VKH or CSCR. As VKH is a diagnosis of exclusion, we decided to do a systemic workup. The patient was empirically treated with oral prednisolone $50 \mathrm{mg}$ daily to avoid loss of time before completing the systemic workup as the visual acuity was deteriorating.

After 4 days, we were informed that the patient had been admitted to a general hospital due to spontaneous ecchymosis and melena. On complete blood count, there was a high white cell count, thrombocytopenia, and low hemoglobin concentration with a probable diagnosis of leukemia. On bone marrow biopsy, ALL with B-cell precursor was confirmed. After antileukemia chemotherapy (ALL induction regimen: cyclophosphamide, vincristine, daunorubicin, 


\section{Case Reports in Ophthalmology}

Case Rep Ophthalmol 2020;11:481-485 DOI: $10.1159 / 000509341$

(c) 2020 The Author(s). Published by S. Karger AG, Basel www.karger.com/cop

Hassanpoor and Niyousha: Acute Lymphoblastic Leukemia Presenting as Acute VogtKoyanagi-Harada Syndrome

L-asparaginase, and prednisolone), the patient's vision was improving, and 2 months later, the patient was on remission. His visual acuity improved to 160/200, and all subretinal fluid was absorbed in both eyes clinically. Patient examination during and after treatment was done in a general hospital by indirect fundoscopy without any other imaging, such as FA and OCT. Unfortunately, the patient expired 10 months after initial presentation.

\section{Discussion}

Based on our literature review, there are few reports of leukemia with an initial presentation as VKH. In a similar case report, a 42-year-old man with bilateral exudative retinal detachment presented with a first diagnosis of $\mathrm{VKH}$, and, later, ALL was confirmed. One week after induction of systemic chemotherapy, visual acuity was recovered [2].

The choroid with its high blood supply can be a suitable tissue for circulating blast cells that can lead to choroidal infiltration with malignant cells. Choriocapillaris occlusion and consequent ischemia can cause retinal pigment epithelial dysfunction. On the other hand, leukemic cells secrete different factors that can cause increased exudation. This increased fluid exudation due to the tumoral nature of these cells and reduced resorption of subretinal fluid due to dysfunction of the retinal pigment epithelium can lead to exudative retinal detachment [2, 3].

In this case, the first differential diagnoses were VKH and CSCR. Nevertheless, due to multiple pinpoint leakages on FA, bilateral involvement, and patient age, the VKH diagnosis was more probable.

An important point that should be noticed by clinicians is that infiltration of leukemic cells in the choroid can lead to an undulating choroidal surface or sick-sea appearance in the choroid that can mimic retinal pigment epithelium undulation, which is an important feature of VKH especially in differentiating it from CSCR [4]. Another important point in leukemia-induced subretinal fluid is that none of the reported cases showed "subretinal septa" on OCT, which is generally evident in typical VKH cases.

Like the case described by Yang and $\mathrm{Yu}$ [2], in our case, on FA, hyperfluorescent dots were surrounding the detached area while sparing the macula (Fig. 1). In VKH, on FA, hyperfluorescent dots are usually spread over the posterior retina, not surrounding the detached area. This sign on FA should be considered as a suspicious pattern for leukemia by clinicians.

Exudative retinal detachment can be a primary presentation of ALL and/or lymphoma. VKH-like presentation can also happen in chronic myeloid leukemia as its first manifestation or even after disease remission [5]. An important criterion for the diagnosis of VKH is the absence of other systemic diseases, and VKH is a diagnosis of exclusion [5]. It is important to keep this in mind - especially in atypical presentations and older age - in order not to miss important life-threating underlying diseases. Leukemia and lymphoma should be considered in the differential diagnosis of VKH in atypical cases, as treatment with systemic corticosteroids and other immunosuppressive agents can change the disease presentation and delay prompt lifesaving treatments [6]. 


\section{Case Reports in Ophthalmology}

\section{Conclusion}

Exudative retinal detachment can be a primary presentation of leukemia, and prompt diagnosis by an ophthalmologist in such cases can result in timely management. To the best of our knowledge, this is the first case of ALL presenting with VKH-like syndrome in Iran.

\section{Statement of Ethics}

The patient's guardians have given their written informed consent to publish the case data and images. The research was conducted ethically in accordance with the Declaration of Helsinki.

\section{Conflict of Interest Statement}

The authors have no conflicts of interest to declare.

\section{Funding Sources}

The study received no grant.

\section{Author Contributions}

Both authors contributed to patient management, data gathering, and manuscript writing. Both authors approved the final version of the manuscript for publication.

\section{References}

1 AlZamil WM. Lymphocytic leukemia presenting as acute Vogt-Koyanagi-Harada disease. Saudi J Ophthalmol. 2014 Oct;28(4):319-21.

2 Yang HK, Yu HG. Acute lymphoblastic leukemia manifesting as acute Vogt-Koyanagi-Harada disease. Korean J Ophthalmol. 2009 Dec;23(4):325-8.

3 Fackler TK, Bearelly S, Odom T, Fekrat S, Cooney MJ. Acute lymphoblastic leukemia presenting as bilateral serous macular detachments. Retina. 2006 Jul-Aug;26(6):710-2.

4 Shields CL, Manalac J, Das C, Saktanasate J, Shields JA. Review of spectral domain enhanced depth imaging optical coherence tomography of tumors of the choroid. Indian J Ophthalmol. $2015 \mathrm{Feb}$;63(2):117-21.

5 Mistry S, Sudharshan S, Ganesan S, Akbar AB, Biswas J. Vogt-Koyanagi-Harada disease like presentation in patients with chronic myeloid leukemia. Am J Ophthalmol Case Rep. 2018 Mar;10:221-5.

6 Smith AG, London NJ, Pao K, Lipkowitz J, Kurup SK, Garg SJ. Leukemia presenting as serous retinal detachment. Retin Cases Brief Rep. 2014;8(4):279-82. 


\section{Case Reports in Ophthalmology}
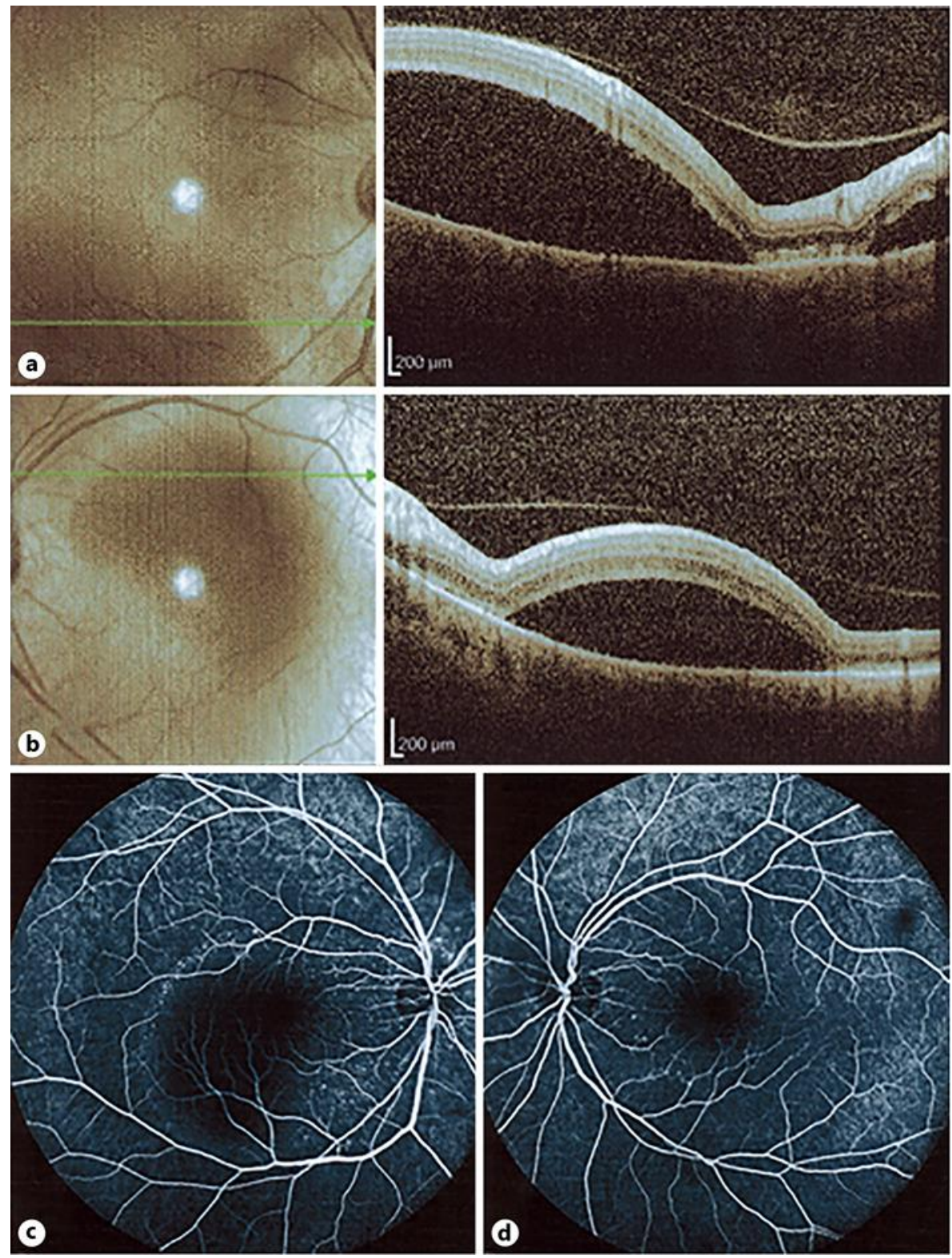

Fig. 1. Subretinal exudative fluid is evident in the right (a) and left (b) eyes on optical coherence tomography at presentation. Fluorescein angiography $(\mathbf{c}, \mathbf{d})$ showed a hypofluorescent area compatible with subretinal fluid and multiple pinpoint hyper- and hypofluorescent dots within the detached retina. Hyperfluorescent dots were distributed mostly in the periphery of the subretinal fluid area while sparing the central macula. 